\title{
Using the Mathematica Computer System on Tylor Derivatives
}

\author{
${ }^{1}$ Anton N. Karamyshev, ${ }^{2}$ Zhanna I. Zaytseva, ${ }^{3}$ Natalia I. Gubochkina \\ ${ }^{1-3}$ Kazan Federal University \\ Email: antonkar2005@yandex.ru
}

Received: $2^{\text {th }}$ August 2019, Accepted: $3^{\text {th }}$ September 2019, Published: $31^{\text {st }}$ October 2019

\begin{abstract}
The ability to find a derivative and use it in different fields plays a large role in the practical activities of students, both in technical specialties, and in economic, and humanitarian ones. The concept of the derivative is one of those mathematical concepts that are intersubjective in nature and are actively used in economic, chemical, physical, and other branches of science and their applications. The study of material on this topic is of great importance since it shows the possibilities of using elements of differential calculus in the description and study of processes, phenomena of the real world. In the school course of algebra and the beginnings of analysis, the concept of a first-order derivative is introduced and the rules of differentiation are derived, in the same place, it is shown in the example of the function $y=|x|$ that it does not have a derivative at zero. In the course of higher mathematics, the concept of derivative expands; the derivative of the $n$th order is understood to mean a function that is the first-order derivative of the derivative of the $(n-1)$ the order. Moreover, there are functions that are continuous at each point of the set of real numbers, but at no point have a derivative. In this regard, the article examines the possibility of applying tayloresque derivative in mathematical education secondary schools in solving academic and applied tasks, and suggested ways of using the computer mathematics system Mathematica as the means for performing computation in the process of solving training tasks in the classroom and to create demonstration and simulation software tools. So, for example, under the topic "the Maclaurin Series", we propose to use tayloresque derivatives and demonstration and simulation software tool created in computer mathematical environment Mathematica, which is based on specific software procedures of the programming language WolframLanguage.
\end{abstract}

\section{Keywords}

Mathematical Analysis, Taylor Derivative, Computer Mathematical System, Computerization of Mathematical Education, Information Technology.

\section{Introduction}

In recent years, there has been a noticeable decline in interest in mathematics from a certain part of pupils and students, and society as a whole, when mathematics is perceived not as an object of professional activity, but as an unnecessary science that will not be useful in future professional activities. Students do not want to spend time and effort to study such a complex, but interesting and exciting subject as mathematics. Therefore, you have to observe that students do not try to delve into the educational process, but increasingly use the Internet to solve their tasks. On the one hand, this has a positive aspect, and on the other, and it is more common, it comes down to profanity, which is called "three clicks", that is, copied - pasted. Such an attitude to mathematics can lead to a loss of computational skills, which is undesirable since it is important for students of engineering fields to solve a mathematical problem to get an answer in the form of an exact or approximate numerical equivalent. Now the teacher, in addition to transferring knowledge, should pay great importance to enhancing the cognitive activity of students, organizing their independent work. In other words, it is necessary to introduce such methods and technologies that are not yet on the Internet and which encourage the student to actively study and develop the ability to independently solve complex mathematical problems.

\section{The Text of the Article}

Consider the concept of the Taylor derivative.

Let $C \equiv C[-1,1]$ be the space of continuous functions on the interval $[-1,1]$ with the norm $\|\varphi\|_{C} \equiv \max _{t \in[-1,1]}|\varphi(t)| \quad(\varphi \in C)$.

The quantities $\varphi^{\{j\}}(0)$ are called the Taylor derivatives of order $\mathrm{j}$ of the function $\varphi \in C$ at the point $t=0$ if the limits exist sequentially [5]:

$$
\begin{gathered}
\lim _{t \rightarrow 0} \frac{\varphi(t)-\sum_{i=0}^{j-1} \varphi^{\{i\}}(0) \cdot t^{i} / i !}{t^{j}}=\varphi^{\{j\}}(0), \\
\left(j=\overline{0, m}, \quad m \in N, \quad \varphi^{\{0\}}(0) \equiv \varphi(0)\right) .
\end{gathered}
$$


Denote by $C\{m ; 0\}$ the class of functions $f(t) \in C$ having at the point $t=0$ the Taylor derivative $f^{\{m\}}(0)$ of order $m$. We assume that $C\{0 ; 0\} \equiv C$ space $C\{m ; 0\}$ is fully and normally embedded in $C$ [1].

If there is an ordinary derivative, then there is a Taylor derivative, and their values at the point are equal. By definition, in this sense, the first-order Taylor derivative coincides with the ordinary derivative.

Some properties of the Taylor derivative are analogs of the known facts from mathematical analysis regarding ordinary derivatives of higher orders [1].

Finding the Taylor derivative of the $m$ th order is sequential and subordinate to a clear algorithm, in each next limit the result of the previous one is used; it's convenient to use electronic resources for this. The use of the Mathematica computer mathematical system is very effective. To calculate the Taylor derivative of a continuous function of one variable, we compiled a program using the WolframLanguage programming language built into this environment. This program formed the basis of another demo-modeling program (DPS), created for the user who will have to work with the dialog box and follow the instructions that appear after each step. The purpose of this demo-modeling program is twofold:

1) it can be used to control calculations performed "manually" (when the essence of the exercise is to consolidate the skills of calculating derivatives),

2) it can be used to automate calculations (when the task of calculating derivatives is included as part of a more complex and relevant task).

In the following example, we will demonstrate both the calculation of the Taylor derivative and the use of the demomodeling software.

Example. Consider the function

$$
\begin{gathered}
y=t^{3} \cdot|t|+16 \cdot t^{2}+7 \cdot t \equiv \varphi(t) . \\
\varphi^{\{0\}}(0)=\varphi(0)=0, \\
\varphi^{\{1\}}(0)=\lim _{t \rightarrow 0} \frac{\varphi(t)-\varphi(0)}{t}=7, \\
\varphi^{\{2\}}(0)=2 ! \lim _{t \rightarrow 0} \frac{\varphi(t)-\varphi(0)-\varphi^{\{1\}}(0) \cdot t}{t^{2}}=32, \\
\varphi^{\{3\}}(0)=3 ! \lim _{t \rightarrow 0} \frac{\varphi(t)-\varphi(0)-\varphi^{\{1\}}(0) \cdot t-\varphi^{\{2\}}(0) \cdot t^{2} / 2 !}{t^{3}}=0, \\
\varphi(t)-\varphi(0)-\varphi^{\{1\}}(0) \cdot t-\varphi^{\{2\}}(0) \cdot t^{2} / 2 !-\varphi^{\{3\}}(0) \cdot t^{3} / 3 ! \\
t^{4}
\end{gathered}
$$

We can conclude: the function in question belongs to space $C\{3 ; 0\}$.

Consider the same example using DMSE in the Mahtematica computer mathematical system to calculate the Taylor derivative, which consists of one hidden cell (Figure 1). In order for the user to apply DMSE, you need to follow the instruction: "Next, select the hidden cell with the left mouse button, press Shift + Enter in turn and follow the further instructions." After this instruction has been fulfilled, a dialog box appears on the screen indicating what exactly needs to be written in it (Figure 1), and then the DMSE enters into a dialogue with the user. 
Then select the hidden cell with the left mouse button, press Shift + Enter and follow the instructions.
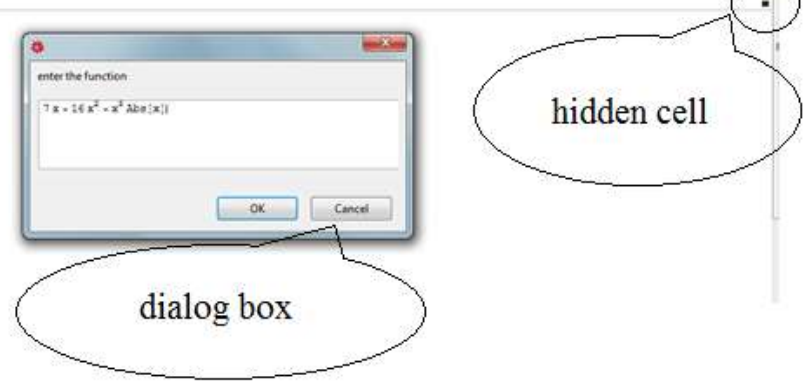

Fig. 1: Working Environment of DMSE for the Derivative

Note that you can enter any function that belongs to this generalized view into the DMSE dialog box. We get the following result (Figure 2).

\section{Then select the hidden cell with the left mouse button, press Shift + Enter and follow the instructions.}

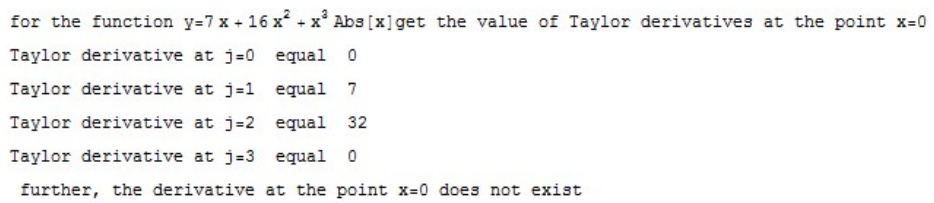

Fig. 2: The Result of the Application of DMSE for the Derivatives

Since one hidden cell is used in DMSE , any (similar) functions belonging to this generalized type can be used in any number, which is important, because, for example, changing the function in the dialog box, you can make sure empirically with the students that the following theorems [1]:

Theorem 1. The function $\varphi(t)$ belongs to the class $C\{m ; 0\}$ if and only if it has the form

$$
\begin{gathered}
\varphi(t)=t^{m} \Phi(t)+\sum_{i=0}^{m-1} a_{i} t^{i}, \\
\text { где } \Phi=T \varphi \in C, a_{i}=\varphi^{\{i\}}(0) / i !,(i=\overline{0, m-1}) .
\end{gathered}
$$

Theorem 2. (an analog of the local Taylor formula). If $\varphi \in C\{m ; 0\}$, then the unique representation is true

$$
\varphi(t)=\sum_{i=0}^{m} \varphi^{\{i\}}(0) t^{i} / i !+o\left(t^{m}\right)(t \rightarrow 0)
$$

Let us conduct a comparative analysis of the application of the ordinary and Taylor derivatives using the example of a fractional rational function from the class of elementary functions. It is such functions that cause dissatisfaction among students for their complex transformations.

Example. Expand the function $f(x)=\frac{x^{2}-x+2}{x^{2}+x+1}$ in the Maclaurin series to members of the fifth order, inclusive.

Solution: The Maclaurin series to members of the fifth-order has the form: 
$f(x)=f(0)+\frac{f^{\prime}(0)}{1 !} x+\frac{f^{\prime \prime}(0)}{2 !} x^{2}+\frac{f^{\prime \prime \prime}(0)}{3 !} x^{3}+\frac{f^{I V}(0)}{4 !} x^{4}+\frac{f^{V}(0)}{5 !} x^{5}+\ldots \ldots \ldots \ldots+R_{n}(x), \quad$ where $R_{n}(x)$ is the residual member of the series.

Here it is necessary to find the value of the function and the values of derivatives up to the fifth-order inclusive at the point $x=0$.

The value of the function at the point $x=0: f(0)=\frac{0^{2}-0+2}{0^{2}+0+1}=2$.

Derivatives are calculated using differentiation rules and a table of derivatives.

The derivative of the first order has the form:

$$
\begin{aligned}
& f^{\prime}(x)=\left(\frac{x^{2}-x+2}{x^{2}+x+1}\right)^{\prime}=\frac{\left(x^{2}-x+2\right)^{\prime} \cdot\left(x^{2}+x+1\right)-\left(x^{2}-x+2\right) \cdot\left(x^{2}+x+1\right)^{\prime}}{\left(x^{2}+x+1\right)^{2}}= \\
& =\frac{(2 x-1) \cdot\left(x^{2}+x+1\right)-\left(x^{2}-x+2\right) \cdot(2 x+1)}{\left(x^{2}+x+1\right)^{2}}=\frac{2 x^{2}-2 x-3}{\left(x^{2}+x+1\right)^{2}},
\end{aligned}
$$

The value of the first-order derivative at the point $x=0: f^{\prime}(0)=\frac{2 \cdot 0^{2}-\cdot 0-3}{\left(0^{2}+0+1\right)^{2}}=-3$,

The second-order derivative has the form:

$$
f^{\prime \prime}(x)=\left(f^{\prime}(x)\right)^{\prime}=-2 \cdot \frac{2 x^{3}-3 x^{2}-9 x-2}{\left(x^{2}+x+1\right)^{3}},
$$

The value of the first-order derivative at the point $x=0: f^{\prime \prime}(0)=4$,

The third-order derivative has the form:

$$
f^{\prime \prime \prime}(x)=\left(f^{\prime \prime}(x)\right)^{\prime}=\frac{6 \cdot\left(2 x^{4}-4 x^{3}-18 x^{2}-8 x+1\right)}{\left(x^{2}+x+1\right)^{4}},
$$

The value of the first-order derivative at the point $x=0: f^{\prime \prime \prime}(0)=6$,

The fourth-order derivative has the form:

$$
f^{I V}(x)=\left(f^{\prime \prime \prime}(x)\right)^{\prime}=-24 \cdot \frac{\left(2 x^{5}-5 x^{4}-30 x^{3}-20 x^{2}+5 x+3\right)}{\left(x^{2}+x+1\right)^{5}},
$$

The value of the fourth-order derivative at the point $x=0: f^{I V}(0)=-72$,

The fifth-order derivative has the form:

$$
f^{V}(x)=\left(f^{I V}(x)\right)=\frac{120 \cdot\left(2 x^{6}-6 x^{5}-45 x^{4}-40 x^{3}+15 x^{2}+18 x+2\right)}{\left(x^{2}+x+1\right)^{6}} \text {, }
$$

The value of the first-order derivative at the point $x=0: f^{V}(0)=240$,

Substitute the Maclaurin series in the formula, we get:

$$
f(x)=2+\frac{(-3)}{1 !} x+\frac{4}{2 !} x^{2}+\frac{6}{3 !} x^{3}+\frac{(-72)}{4 !} x^{4}+\frac{240}{5 !} x^{5}+\ldots \ldots \ldots \ldots+R_{n}(x) .
$$

Thus, the Maclaurin series of the function $f(x)=\frac{x^{2}-x+2}{x^{2}+x+1}$ to members of the fifth-order has the form:

$$
f(x)=2-3 x+2 x^{2}+x^{3}-3 x^{4}+2 x^{5}+\ldots \ldots \ldots \ldots+R_{n}(x) .
$$

Now, for the same function, we find the values of derivatives up to the fifth-order inclusive at the point $x=0$, using the Taylor derivative.

The value of the function at the point $x=0: f^{\{0\}}(0)=f(0)=2$. 
The value of the first-order derivative at the point $\mathrm{x}=0$ :

$$
f^{\{1\}}(0)=\lim _{x \rightarrow 0} \frac{f(x)-f(0)}{x}=\lim _{x \rightarrow 0} \frac{\frac{x^{2}-x+2}{x^{2}+x+1}-2}{x}=\lim _{x \rightarrow 0} \frac{-x-3}{x^{2}+x+1}=-3,
$$

The value of the first-order derivative at the point $\mathrm{x}=0$ :

$$
f^{\{2\}}(0)=2 ! \lim _{x \rightarrow 0} \frac{f(x)-f(0)-f^{\{1\}}(0) \cdot x}{x^{2}}=2 ! \lim _{x \rightarrow 0} \frac{3 x+2}{x^{2}+x+1}=4,
$$

The value of the first-order derivative at the point $\mathrm{x}=0$ : ,

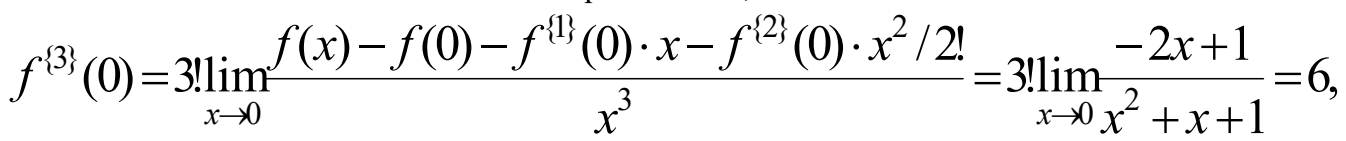

The value of the first-order derivative at the point $\mathrm{x}=0$ :

$$
\begin{aligned}
& f^{\{4\}}(0)=4 ! \lim _{x \rightarrow 0} \frac{f(x)-f(0)-f^{\{1\}}(0) \cdot x-f^{\{2\}}(0) \cdot x^{2} / 2 !-f^{\{3\}}(0) \cdot x^{3} / 3 !}{x^{4}}= \\
& =4 ! \lim _{x \rightarrow 0} \frac{-x-3}{x^{2}+x+1}=-72
\end{aligned}
$$

The value of the first-order derivative at the point $\mathrm{x}=0$ :

$$
\begin{aligned}
& f^{\{5\}}(0)=5 ! \lim _{x \rightarrow 0} \frac{f(x)-f(0)-f^{\{1\}}(0) \cdot x-f^{\{2\}}(0) \cdot x^{2} / 2 !-f^{\{3\}}(0) \cdot x^{3} / 3 !-f^{\{4\}}(0) \cdot x^{4} / 4 !}{x^{5}}= \\
& =5 ! \lim _{x \rightarrow 0} \frac{3 x+2}{x^{2}+x+1}=240 \ldots
\end{aligned}
$$

Substitute the Maclaurin series in the formula and get the same result:

$$
f(x)=2-3 x+2 x^{2}+x^{3}-3 x^{4}+2 x^{5}+\ldots \ldots \ldots \ldots+R_{n}(x) .
$$

Obviously, calculating the values of derivatives at a point using Taylor derivatives frees from routine, cumbersome calculations, which makes them attractive for use in the educational process, as well as for solving some specific mathematical problems by students of technical specialties. Acting by definition, bypassing the rules of differentiation, the student more deeply comprehends the concept of a derivative as the limit of the ratio of the increment of a function to the increment of an argument when the latter tends to zero.

It is also possible to effectively use the DEM program in the classroom, created in the Mathematica computer mathematical environment (Figure 3) to calculate the coefficients of the Maclaurin series, which consists of one hidden cell (Figure 3). In order for the user to apply DMSE, you need to follow the instruction: "Next, select the hidden cell with the left mouse button, press Shift + Enter in turn and follow the further instructions." After that, a dialog box appears on the screen with the inscription what exactly needs to be entered in the calculation task cell, and the DMSE enters a dialogue with the user.

Then select the hidden cell with the left mouse button, press Shift + Enter and follow the instructions.

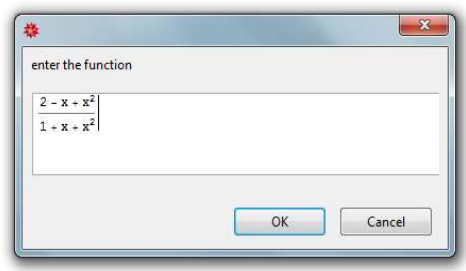

Fig. 3: Operating Medium of DMSE for the Maclaurin Series 
Note that since a single hidden cell is used in DMSE, any elementary function can be entered into the dialog box (Figure 3). We get the following result (Figure 2).

Then select the hidden cell with the left mouse button, press Shift + Enter and follow the instructions.

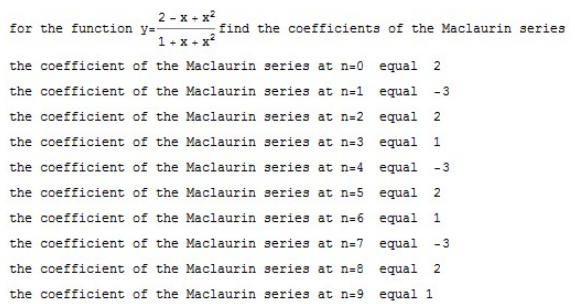

Fig. 4: DMSE Result for the Maclaurin Series

It is very clearly seen that for the function we introduced, the coefficient values are repeated (Figure 4), and due to the cumbersomeness of the calculations, we cannot find the derivative of the order and its value at the point $x=0$, but we can find any coefficient a series or value of a derivative of any order; for example, we find the value of the 50-order derivative:

$$
a_{50}=\frac{f^{(50)}(0)}{50 !}=a_{3 \cdot 16+2}=a_{2}=2,
$$

we get in this case that $f^{(50)}(0)=50 ! \cdot 2$.

\section{Methods}

We used the following methods:

1. theoretical - analysis of scientific literature on mathematical, methodological and special aspects related to this topic;

2. empirical - conversations, pedagogical observations, questioning of students and teachers;

3. programming methods in the WolframLanguage language.

\section{Results and Discussion}

The basic techniques and methods of introducing into the educational process of the university the Taylor derivative function of one variable and the Mathematica computer mathematical system are formulated.

A demo-modeling software tool (DMPS) was developed and compiled on the basis of specific software procedures of the WolframLanguage programming language in the Mathematica computer mathematical environment on the topics "Taylor derivative of a function of one variable" and "Maclaurin series" in a single format for user convenience.

The methods of introducing the Taylor derivative function of one variable into the educational process have not been considered in previous studies.

\section{Summary}

The use of a single variable in the Taylor derivative function of a function using the demo-modeling software used for this, created in the Mathematica computer environment, greatly simplifies the computational process and makes the process of teaching mathematics fascinating; this gives us the opportunity to conduct research work, since we can take for consideration any elementary function and do a lot of calculations in a short time. Thus, we not only calculate but also make subjectively new (for students) scientific mathematical conclusions.

\section{Conclusions}

The use of the Taylor derivative function of one variable and the Mathematica computer mathematical system in teaching allows you to intensify the educational process, reducing time spent on laborious but completely routine calculations, increasing the level of visibility and the level of information, involving students in the research process, instilling a taste for mathematical experiments. Informatization of the process of teaching mathematics enriches it with new means.

\section{Acknowledgments}

The work is performed according to the Russian Government Program of Competitive Growth of Kazan Federal University. 


\section{References}

[1] N.S. Gabbasov. Methods for solving Fredholm integral equations in the space of generalized functions. - Kazan, 2006.- 176 pages.

[2] J.I. Zaitseva. Methods of teaching higher mathematics using new information technologies (at a technical university): dis. ... cand. ped Sciences: 13.00.02, 13.00.08; is protected at the Moscow State Educational Institution 12/27/05 / Zh.I. Zaitseva. Elabuga State Pedagogical University - Elabuga, 2005. -140 pages.

[3] J.I. Zaitseva. The use of computer technology in the mathematical education of students of technical specialties // Kazan Science. No. 3 2016. - Kazan: Publishing House of the Kazan Publishing House, 2016.-134 - pp. 108-111.

[4] J.I. Zaitseva, N.I. Gubochkina. The use of the Taylor derivative in the educational process // Kazan Science. No. 3 2017. - Kazan: Kazan Publishing House, 2017.-114 - pp. 75-78.

[5] Z.A. Pressdorrf. Singular integral equation with a symbol that vanishes at a finite number of points // Mat. Research.-Chisinau, 1972.-T7, issue 1. - pp.116-132.

[6] I.V. Robert. (2010) Modern information technologies in education: didactic problems; prospects for use. Moscow, M: IIO RAO.

[7] N.S. Gabbasov, R.R. Zamaliev. Special variants of collocation method for integral equations in a singular case // Russian Mathematics. - 2017. - Vol.61, Is.5. - P.38-45.

[8] N.S. Gabbasov, R.R. Zamaliev. A special variant of the subdomain method for integral equations of the third kind with singularities in the kernel // Russian Mathematics. - 2014. - Vol.58, Is.10. - pp.15-21.

[9] T.V. Kapustina, A.V. Popyrin, and L.N. Savina. (2014).Statistics and Econometrics from the Point of View Methodology Mathematics. World Applied Sciences Journal 31 (6): 1168-1172, doi: 10.5829 / idosi.wasj.2014.31.06.06.14346.

[10] T.V. Kapustina, A.V. Popyrin, and L.N. Savina. (2015). Computer support of interdisciplinary communication of analytic geometry and algebra. International Electronic Journal of Mathematics EducationVolume 10 (3), pp. 177-187, doi: 10.12973 / mathedu.2015.113 pages.

[11] S. Wolfram. (2003). TheMathematicaBook. " Fifth Edition. Mathematica Version 5. - Wolfram Media / Cambridge University Press, 2003. - 1301 pages.

[12] S. Wolfram. (2009). MathematicaDocumentationCenter. Wolfram Research, Date Views 03/07/14 reference.wolfram.com/mathematica/guide/Mathematica.html. 